\title{
The Onset of Dynamic Stall Revisited
}

\author{
Karen Mulleners • Markus Raffel
}

Received: date / Accepted: date

\begin{abstract}
Dynamic stall on a helicopter rotor blade comprises a series of complex aerodynamic phenomena in response to the unsteady change of the blade's angle of attack. It is accompanied by a lift overshoot and delayed massive flow separation with respect to static stall. The classical hallmark of the dynamic stall phenomenon is the dynamic stall vortex. The flow over an oscillating OA209 airfoil under dynamic stall conditions was investigated by means of unsteady surface pressure measurements and time-resolved particle image velocimetry. The characteristic features of the unsteady flow field were identified and analysed utilising different coherent structure identification methods. An Eulerian and a Lagrangian procedure were adopted to locate the axes of vortices and the edges of Lagrangian coherent structures, respectively; a proper orthogonal decomposition of the velocity field revealed the energetically dominant coherent flow patterns and their temporal evolution. Based on the complementary information obtained by these methods the dynamics and interaction of vortical structures were analysed within a single dynamic stall life cycle leading to a classification of the unsteady flow development into five successive stages: the attached flow stage; the stall development stage; stall onset; the stalled stage; and flow reattachment. The onset of dynamic stall was specified here based on a characteristic mode of the proper orthogonal decomposition of the velocity field. Variations in the flow field topology that accompany the stall onset were verified by the Lagrangian coherent structure analysis. The instantaneous effective unsteadiness was
\end{abstract}

K. Mulleners ( ) M. Raffel

German Aerospace Center (DLR),

Bunsenstr. 10, 37073 Göttingen, Germany

E-mail: karen.mulleners@dlr.de defined as a single representative parameter to describe the influence of the motion parameters. Dynamic stall onset was found to be promoted by increasing unsteadiness. The mechanism that results in the detachment of the dynamic stall vortex from the airfoil was identified as vortex induced separation caused by strong viscous interactions. Finally, a revised criterion to discern between light and deep dynamic stall was formulated.

Keywords dynamic stall · time-resolved PIV . coherent structure analysis · unsteady separation

\section{List of Symbols} $a_{\mathrm{i}}$

$c$

$C_{1}$

$C_{\mathrm{m}}$

$C_{\mathrm{p}}$

$f_{\text {osc }}$

$k$

$M a$

$M$

$n$

N

\section{$\mathbf{n}$}

$r_{\text {eq }}$

Re

$S$

$t_{\mathrm{n}}$

$t_{\mathrm{ss}}$

$T$

$\mathbf{u}=(u, v, w)$

$U_{\infty}$

$\mathbf{x}=(x, y, z)$

$\alpha$

$\alpha_{0}$

$\alpha_{1}$ i-th temporal eigenmode of the POD

chord length

lift coefficient

pitching moment coefficient

pressure coefficient

oscillation frequency

reduced frequency

Mach number

number of grid points

probability density

number of PIV velocity fields

unit normal vector

reduced pitch rate

Reynolds number

two-dimensional area

discrete time stamp

time at which static stall angle is exceeded

period of oscillation

local velocity

free steam velocity

spatial coordinates

angle of attack

mean incidence

oscillation amplitude 


$\begin{array}{ll}\alpha_{\mathrm{ds}} & \text { dynamic stall angle of attack } \\ \alpha_{\max } & \text { maximum angle of attack } \\ \alpha_{\mathrm{ss}} & \text { static stall angle of attack } \\ \dot{\alpha}_{\mathrm{ss}} & \text { instantaneous effective unsteadiness } \\ \Gamma & \text { scalar function } \\ \Delta t_{\mathrm{ds}} & \text { dynamic stall delay } \\ \lambda_{\mathrm{i}} & \text { i-th eigenvalue of the POD } \\ \theta & \text { velocity orientation angle } \\ \omega & \text { vorticity } \\ \psi_{\mathrm{i}} & \text { i-th spatial eigenmode of the POD } \\ \nearrow & \text { during upstroke } \\ \searrow & \text { during downstroke } \\ O & \text { order of magnitude }\end{array}$

\section{Introduction}

The dynamic stall process of the flow over a constantly pitching airfoil, a classic example of unsteady separation, comprises a series of complex aerodynamic phenomena. It features a lift overshoot and a delay in the onset of massive flow separation with respect to static stall. The salient feature of the unsteady separating flow is the formation and convection of a large-scale coherent structure referred to as the dynamic stall vortex. The most prominent example can be observed on the retreating blades of a helicopter rotor in forward flight. Although the dynamic stall delay and the related increase of the maximum lift are convenient in some applications, the large excursions of the aerodynamic loads during vortex break down are adverse to helicopter rotors as strong vibrations and potentially fatal structural loads are introduced which are potentially fatal for a helicopter rotor. Due to the incessant interest in improving the manoeuvrability and performance of rotary-wing aircraft and rapidly manoeuvring aircraft, dynamic stall has been the subject of numerous investigations during past decades (McAlister et al 1978; McCroskey 1981; Carr 1988).

The analysis of dynamic stall events on an oscillating airfoil by Carr et al (1977) revealed that the prominent features within a full cycle of oscillation are consecutively the emergence and spreading of flow reversal on the airfoil's suction side, the formation and convection of a large-scale leading edge vortex, massive flow separation, and flow reattachment. Analogously, Shih et al (1992) classified the unsteady flow development over an airfoil pitching up at constant rate into four successive stages: a vortex formation stage, a vortex convection stage, stall onset, and a stalled stage. Both descriptions show that the flow over either a constantly pitching or oscillating airfoil is qualitatively described by the same characteristic features, being the initiation, growth and shedding of a leading edge vortex and the associated lift overshoot. For both types of motion the process of vortex formation and convection result in a delay of massive flow separation to angles of attack beyond the static stall angle. During this delay the lift continues to increase with increasing angle of attack yielding the characteristic lift overshoot. The inception of stall is generally accompanied by a loss of lift and an increase of the negative pitching moment and marks the beginning of the stalled stage. This stage is recognised by large-scale vortex shedding and associated large fluctuations of the lift, drag and pitching moment. When the airfoil motion is oscillatory the airloads show large hysteresis.

Despite extensive analytical, numerical and experimental efforts, the phenomenology of dynamic stall is not yet fully understood and characterised. In particular, the process that leads to the formation of the primary stall vortex and the mechanism that causes the vortex to detach are still controversial. Additional investigations are required to provide a reliable assessment of the dynamic stall onset, which is mandatory for successful dynamic stall modelling and prediction. Possible indicators of stall onset are summarised by Sheng et al (2006) and include the point at which the pitching moment coefficient breaks, a deviation in the lift or the drag coefficient occurs, and the maximum or critical leading edge suction is achieved (Wilby 2001; Leishman and Beddoes 1989). These criteria are all based on the examination of airloads and a direct correlation with coherent or vortical structures in the flow field is still pending. A new method to identify stall onset based directly on the flow field is required.

Accurate knowledge of the flow field during dynamic stall is strongly tied to a fundamental understanding of the development and interaction of coherent structures. Only recently time--resolved particle image velocimetry (TR-PIV) became applicable which allows for analysis of the spatiotemporal evolution of the velocity field in general and coherent structures in particular. However, experimental investigation of vortex dynamics and interactions remains challenging due to the lack of a universally accepted definition of a vortex. Several definitions have been proposed hitherto (see e.g. Lugt 1979; Robinson 1991; Haller 2005), but no consensus has been reached yet. As a direct consequence, unambiguous vortex detection remains elusive and various Eulerian and Lagrangian criteria have been introduced whose adequateness depends on the specific problem at hand (Cucitore et al 1999; Chakraborty et al 2005).

Within the scope of this study, the conspicuous features of the experimentally investigated flow over a sinusoidally oscillating airfoil in a uniform flow are iden- 
tified and analysed utilising a combination of an Eulerian vortex centre allocation procedure (Michard et al 1997), a Lagrangian approach based on the Lyapunov exponent (Haller 2001), and a proper orthogonal decomposition (POD) of the velocity field (Sirovich 1987). Whereas past experimental investigations generally involve phase-locked measurements, the present study provides time-resolved velocity field information in addition to unsteady surface pressure distributions. This allows for the examination of the chronology of events during a single cycle of oscillation. The main objective is to combine the different vortex identification procedures to analyse the spatiotemporal evolution of vortical structures within the dynamic stall life cycle and to specify directly the onset of dynamic stall.

The paper is organised as follows. Prior to the description of the applied coherent structure identification procedures, the experimental methods will be specified. Subsequently, the experimental results are presented and discussed covering the examination of the chronology of events during an entire dynamic stall life cycle and a detailed analysis of the onset of dynamic stall. The discussion is concluded by a short summary of the most important findings and by suggesting further avenues of investigation.

\section{Experimental Set-up}

Wind tunnel experiments were conducted to investigate the dynamic stall life cycle on a constantly pitching airfoil in a uniform flow at a free stream Reynolds number $R e=9.2 \times 10^{5}$ based on the chord length $c$ (Mach number $M a=0.14$ ). A two-dimensional airfoil model with an OA209 profile was subjected to a sinusoidally oscillating motion about its quarter chord axis with a mean incidence $\alpha_{0}$, an amplitude $\alpha_{1}$, and an oscillation frequency $f_{\text {osc }}$. The latter is preferably written in dimensionless form as the reduced frequency $k=\pi f_{\text {osc }} c / U_{\infty}$, where $U_{\infty}$ is the free steam velocity. The mean incidence, amplitude and reduced frequency were varied such that $\alpha_{0} \in\left\{18^{\circ}, 20^{\circ}, 22^{\circ}\right\}, \alpha_{1} \in\left\{6^{\circ}, 8^{\circ}\right\}$, and $k \in\{0.050,0.075,0.10\}$.

Stereoscopic TR-PIV was conducted in the crosssectional plane at model mid-span. The width of the field of view covered the entire chord for the relevant angle of attack range. Time series of 6144 frames at full camera resolution (i. e. $1024 \mathrm{px} \times 1024 \mathrm{px}$ ) were recorded at $3000 \mathrm{~Hz}$, corresponding to an acquisition rate of $1500 \mathrm{~Hz}$ for the velocity fields. After mapping the views of both cameras, the dimensions of the PIV measurement window were $335 \mathrm{~mm} \times 165 \mathrm{~mm}$ with a spatial resolution of $5.0 \mathrm{px} / \mathrm{mm}$. The PIV images were processed using an interrogation window size of $32 \mathrm{px} \times 32 \mathrm{px}$ and an overlap of approximately $80 \%$ yielding a grid spacing of $6 \mathrm{px}$ or $1.2 \mathrm{~mm}$ which is less then $0.005 \mathrm{c}$. The interrogation window size was minimised ensuring an acceptable signal-to-noise ratio. The window overlap on the other hand was maximised to avoid artificial smoothing of velocity gradients (Richard et al 2006). By doing so the spatial resolution of the results of the vortex detection algorithms was improved. Prior to the coherent structure analysis, the velocity fields were rotated into the airfoil reference system with the $x$-axis along the chord, the $y$-axis along the span and the $z$-axis upward perpendicular to the chord, while the origin coincides with the rotation axis, i. e. the airfoil's quarter chord axis, at model mid-span. Simultaneously to the TR-PIV, the surface pressure distribution at the model mid-span was scanned at approximately $6 \mathrm{kHz}$ for about $15 \mathrm{~s}$. The data acquisition was synchronised with the recording of the PIV images allowing for straightforward assignment of the instantaneous pressure distributions to each of the acquired velocity fields.

\section{Coherent Structure Analysis}

The common goal of coherent structure identification methods is to locate, extract, and visualise flow structures that are characterised by various spatial and temporal scales.

\subsection{Eulerian Method}

A literature survey yields a considerable number of Eulerian identification criteria. Eulerian structure detection usually deals with spatial concentrations of quantities derived from the instantaneous velocity field and its gradients. Comprehensive reviews of the diversity of Eulerian vortex identification schemes and their applications are provided by Jeong and Hussain (1995) and Wu et al (2006). Among the most commonly used Eulerian vortex detection criteria are the $Q$-criterion introduced by Hunt et al (1988), the $\Delta$-criterion of Chong et al (1990), and the $\lambda_{2}$-criterion proposed by Jeong and Hussain (1995). In most flow situations these three criteria yield similar structures which correctly represent the topology and geometry of the vortex cores in the Eulerian frame of reference. Nevertheless, they share several disadvantages.

An important disadvantage of gradient-based criteria is their susceptibility to measurement noise, rendering them inadequate for application on experimental data, such as instantaneous velocity fields measured with PIV. Due to numerical differentiation measurement noise can severely contaminate the derivatives 

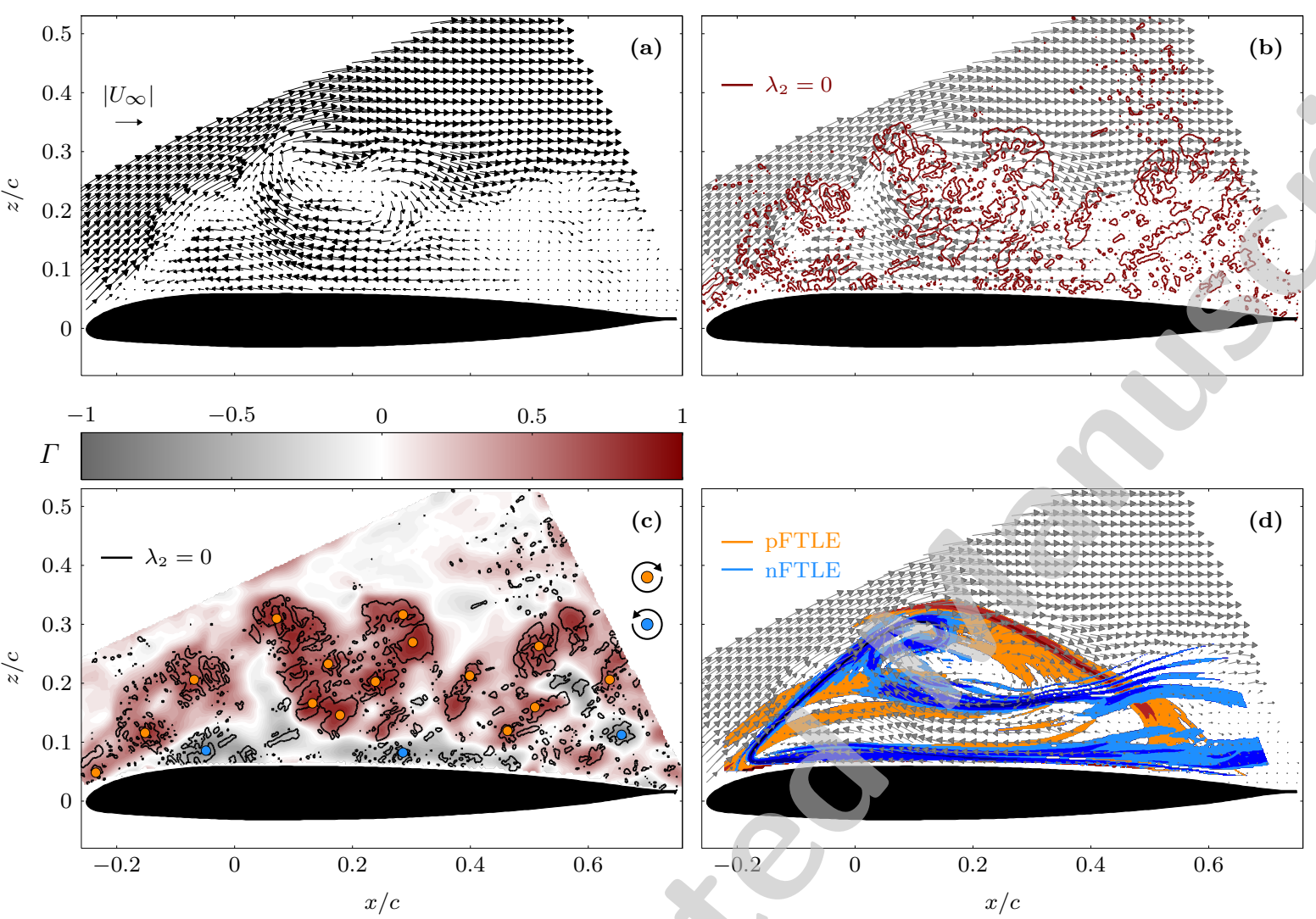

Fig. 1 (a) The instantaneous velocity field at $\alpha=26.8^{\circ}$ on the upstroke $\left(\alpha_{0}=20^{\circ}, \alpha_{1}=8^{\circ}, k=0.050\right)$; Eulerian vortex detection: (b) the corresponding zero contour lines of $\lambda_{2}$ and (c) a combined representation of the scalar field $\Gamma$ (colour-coded) and the zero contour lines of $\lambda_{2}$, the markers indicate the location of $(\bullet)$ clockwise and $(\bullet)$ anticlockwise rotating vortices determined by the $\Gamma$-criterion; (d) Lagrangian coherent structures indicated by the ridges in the PFTLE and nFTLE fields.

yielding less reliable vortex core identification. This is elucidated in figure 1 for the $\lambda_{2}$-criterion; Jeong and Hussain (1995) postulated that vortex cores are regions where $\lambda_{2}<0$. Hence, the presence and location of vortical structures in the instantaneous velocity field depicted in figure $1(\mathrm{a})$ is revealed by the zero contour lines of $\lambda_{2}$ (figure $1(\mathrm{~b})$ ). Although the $\lambda_{2}$-identification scheme seems able to discern single structures in the shear zone between the viscous separated flow region and the inviscid external flow, it does neither allow to pinpoint the individual vortex axes nor to determine the geometry of the various vortex cores. This clearly reveals the need for an alternative, preferably non-local, Galilean invariant procedure which does not require the computation of derivatives. The alternative solution that was adopted here is based on a two-dimensional form of the dimensionless scalar function $\Gamma$ (introduced by Michard et al 1997). The function is derived directly from the two-dimensional in-plane velocity field and is defined in discrete form as

$$
\begin{aligned}
\Gamma\left(\mathbf{x}_{\mathrm{i}}\right) & =\frac{1}{M} \sum_{\mathbf{x}_{\mathrm{j}} \in S_{\mathrm{i}}} \frac{\left[\left(\mathbf{x}_{\mathrm{j}}-\mathbf{x}_{\mathrm{i}}\right) \times\left(\mathbf{u}_{\mathrm{j}}-\tilde{\mathbf{u}}_{\mathrm{i}}\right)\right] \cdot \mathbf{n}}{\left|\mathbf{x}_{\mathrm{j}}-\mathbf{x}_{\mathrm{i}}\right| \cdot\left|\mathbf{u}_{\mathrm{j}}-\tilde{\mathbf{u}}_{\mathrm{i}}\right|} \\
& =\frac{1}{M} \sum_{\mathbf{x}_{\mathrm{j}} \in S_{\mathrm{i}}} \sin \left(\theta_{\mathrm{ij}}\right)
\end{aligned}
$$

with $S_{\mathrm{i}}$ a two-dimensional area around $\mathbf{x}_{\mathrm{i}}, M$ the number of grid points $\mathbf{x}_{\mathbf{j}}$ inside $S_{\mathrm{i}}$ with $j \neq i, \mathbf{n}$ the unit normal vector, $\mathbf{u}_{\mathrm{j}}$ the velocity at $\mathbf{x}_{\mathrm{j}}, \tilde{\mathbf{u}}_{\mathrm{i}}$ the local mean velocity around $\mathbf{x}_{\mathbf{i}}$, and $\theta_{\mathrm{ij}}$ the angle formed by the vectors $\mathbf{x}_{\mathrm{j}}-\mathbf{x}_{\mathrm{i}}$ and $\mathbf{u}_{\mathrm{j}}-\tilde{\mathbf{u}}_{\mathrm{i}}$. The local mean velocity is taken into account in order for $\Gamma$ to be Galilean invariant (cf. Graftieaux et al (2001)).

According to its definition, $\Gamma$ is a dimensionless scalar function, with $-1 \leq \Gamma \leq 1$. The location of possible vortex axes is indicated by the local extrema of $\Gamma$ and the sense of rotation is given by the sign of the extrema. The distribution of $\Gamma(\mathbf{x})$ is computed for the instantaneous velocity field depicted in figure 1(a) and is colour-coded in figure 1(c), where the markers 
indicate the assessed vortex axes locations. The zero contour line of $\lambda_{2}$ allows for the validation of the detected vortex centres.

Besides the detection of the location of the vortex centres, their trajectories over a time series of flow fields are traced. For this purpose, the convection velocity of the individual identified vortex centres within the reference frame are used to predict their future position and narrow the number of possible follow-up vortices. The tracing of the trajectories allows for the investigation of the spatiotemporal evolution of the vortical structures.

\subsection{Lagrangian Approach}

Alternatively to the routinely used Eulerian methods, coherent structure identification algorithms that are Lagrangian in nature have been introduced recently into the fluid dynamics community (Peacock and Dabiri 2010, and references therein). The Lagrangian approach leverages the properties of fluid particle trajectories for the identification of coherent structures. Hence, Lagrangian vortex detection methods are inherently objective, they include information on the history of the flow, and they have a clear physical interpretation. The most popular Lagrangian approach, which was adopted in the present study, is based on finite-time Lyapunov exponents (FTLE) (Haller 2001).

The FTLE method reverts directly to the fluid particle trajectories which can be integrated forward and backward in time yielding positive and negative finitetime Lyapunov exponent (pFTLE and nFTLE) fields (see e.g. Haller 2002; Shadden et al 2005; Garth et al 2007 , for a comprehensive discussion of the general properties and basic concepts involved in the computation of the FTLE).

The ridges in the pFTLE field reveal material lines normal to which fluid particles are being stretched or repelled, consequentially they are referred to as repelling material lines or stable manifolds. Vice versa, ridges in nFTLE fields visualise attracting materials or unstable manifolds, i. e. lines along which fluid particles are being elongated, when integrating the trajectories in backward time. The flow field around the intersection of a repelling and an attracting material line resembles that of a saddle point. Moreover, when attached to a solid surface attracting material lines depict separation lines while attachment lines are repelling material lines. This FTLE method thus yields candidate material lines and captures features of the flow that are familiar from flow visualisation experiments. According to Shadden et al (2005) the ridges in the FTLE fields delineate regions that exhibit qualitatively different dynamical be- haviour, hence indicated the boundaries of Lagrangian coherent structures (LCSs).

The presence and form of the relevant LCSs corresponding to the previously presented instantaneous velocity field are indicated in figure $1(\mathrm{~d})$ by the ridges in the pFTLE and nFTLE fields that are blank for values less than $30 \%$ of the maximum of the field. The FTLE fields presented and discussed in the course of this paper have been computed based on the two-dimensional inplane velocity field utilising the software package ManGen created by Lekien and Coulliette (2001-2002).

The FTLE-method is robust and relatively insensitive to short term anomalies in the velocity data and is particularly suited to analyse experimental data (Haller 2002). Applying the FTLE-method on the original set of measured velocity fields yields a complex network of LCSs and critical points, especially for fluid flows of moderate and high Reynolds number. A low-order POD reconstruction was adopted (Berkooz et al 1993) to filter and smoothen the data set prior to the Lagrangian analysis. According to the basic properties of the POD, the application of the FTLE method on a set of low-order reconstructed fields allows to focus on the dynamics and the topological signature of the largescale coherent structures that dominated the flow field. The prominent critical points of the LCS topology are revealed more clearly, and crucial events, such as vortex interaction and detachment, can be detected at their earliest stage. All FTLE fields depicted here have been calculated based on the low-order POD reconstruction of the velocity field retaining the first 10 modes. The Eulerian method was applied directly on the measured instantaneous velocity fields.

\subsection{Proper Orthogonal Decomposition}

A third approach to extract flow structures is based on a POD of the flow field. The POD method denotes a procedure for finding a basis of orthogonal spatial and temporal functions for a modal bi-orthogonal decomposition from an ensemble of spatiotemporal signals (Aubry et al 1991). The fundamental idea is to represent the random spatiotemporal signal as a series of the deterministic spatial functions with the temporal functions as random coefficients such that the original signal is approximated as accurate as possible based on an energy-weighted measure.

With regard to the present investigation, the twodimensional in-plane velocity field $\mathbf{u}=(u, w)$ was decomposed according to

$\mathbf{u}\left(x, z, t_{\mathrm{n}}\right)=\sum_{i=1}^{N} a_{\mathrm{i}}\left(t_{\mathrm{n}}\right) \psi_{\mathrm{i}}(x, z) \quad$, 
where $N$ is the number of instantaneous field realisations and $t_{\mathrm{n}}$ is the discrete time stamp. According to Lumley (1970), every spatial function or mode $\psi_{\mathrm{i}}(x, z)$ can be associated with an instantaneous organised flow pattern whose temporal evolution is described by the corresponding temporal mode $a_{\mathrm{i}}(t)$ and whose relative contribution to the total energy is represented by the corresponding eigenvalue $\lambda_{\mathrm{i}}$. The eigenvalues are sorted in decreasing order such that the corresponding first modes represent the most dominant flow structures in terms of energy content.

Thus, the POD yields a systematic mathematical tool to define and identify coherent structures in a complex flow system as well as to study their spatiotemporal evolution. The discretised implementation is based on the snapshot method introduced by Sirovich (1987).

\section{Results and Discussion}

Corroborated by the complementary information provided by the different coherent structures identification methods, the unsteady flow development over an oscillation airfoil was divided into five different stages, analogous to the classification of Shih et al (1992) with regard to the flow over an airfoil pitching-up at constant rate. With the starting point of a cycle taken at the minimum incidence angle, the flow will consecutively pass through the following stages within each individual cycle: 1) the attached flow stage; 2) the stall development stage; 3) stall onset; 4) the stalled stage; and 5) flow reattachment.

\subsection{Dynamic Stall Life Cycle}

Attempting a general description of the characteristic features of the dynamic stall life cycle, the velocity field data and surface pressure distributions acquired within a sole harmonic oscillation specified by $\alpha_{0}=20^{\circ}$, $\alpha_{1}=8^{\circ}$, and $k=0.10$ are discussed. The typical dynamic stall curves of the lift and pitching moment coefficients are depicted in figure 2. Additionally, the response of the aerodynamic load coefficients in absence of dynamic effects is presented, revealing a static stall angle of attack $\alpha_{\mathrm{ss}}=21.4^{\circ}$. For selected phase angles the instantaneous velocity fields and surface pressure distributions are depicted in figure 3 together with the locations of vortices determined by the Eulerian detection algorithm.

During the first part of the cycle, i. e. from the minimum incidence angle upstroke to the static stall angle, the flow is attached to the airfoil's surface and the
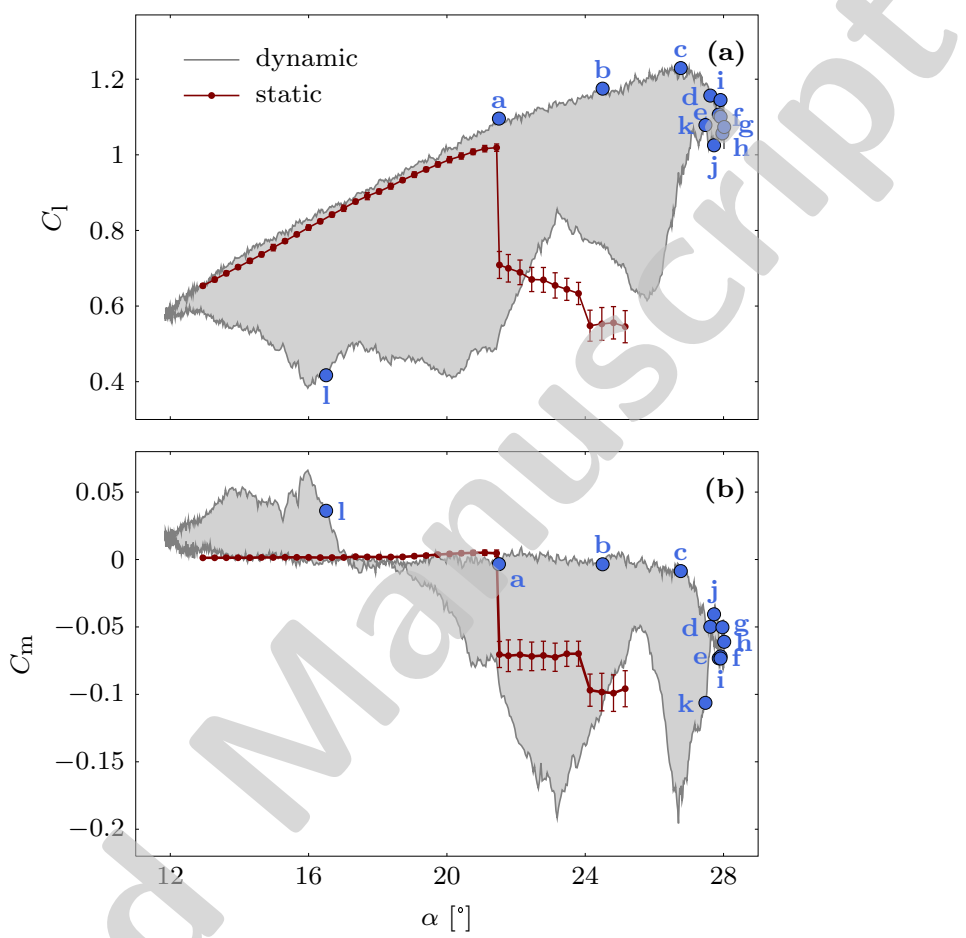

Fig. 2 Static and dynamics airloads as a function of angle of attack: (a) lift and (b) pitching moment coefficient $\left(\alpha_{0}=20^{\circ}\right.$, $\left.\alpha_{1}=8^{\circ}, k=0.10\right)$. The error bars indicate the standard deviation of the static load coefficients. The markers on the dynamic hysteresis curves indicate selected points for which the instantaneous velocity fields and surface pressure distributions are depicted in figure 3 .

surface pressure distribution exhibits an increasing suction peak near the leading edge. Furthermore, the lift increases linearly with the angle of incidence at a rate approximately equal to its static counterpart.

Increasing the angle of attack beyond the static stall angle, an adverse pressure gradient builds up downstream of the leading edge eliciting the development of recirculating flow on the airfoil's suction side. Between this region of flow reversal and the free stream flow a shear layer forms. Shortly after its development, the shear layer is subjected a primary instability (cf. Ho and Huerre 1984) as a result of which the initially contained vorticity is redistributed into individual lumped vortices (figure 3(a)-(c)). At first, these small-scale regularly spaced shear layer vortices - which are all clockwise rotating - are convected downsteam by the external flow and interact only weakly with each other. Meanwhile, the suction peak continues to rise; the lift force increases steadily too, though more slowly than below the static stall angle. The latter is due to the reversing flow layer extending over a considerable part of the airfoil's chord. Hence, despite the occurrence of 


\begin{tabular}{llllllllll}
\multicolumn{1}{l}{-0.04} & -0.03 & -0.02 & -0.01 & 0 & 0.01 & 0.02 & 0.03 & 0.04 \\
$\omega c / U_{\infty}$ & & & & & & & & & \\
\end{tabular}
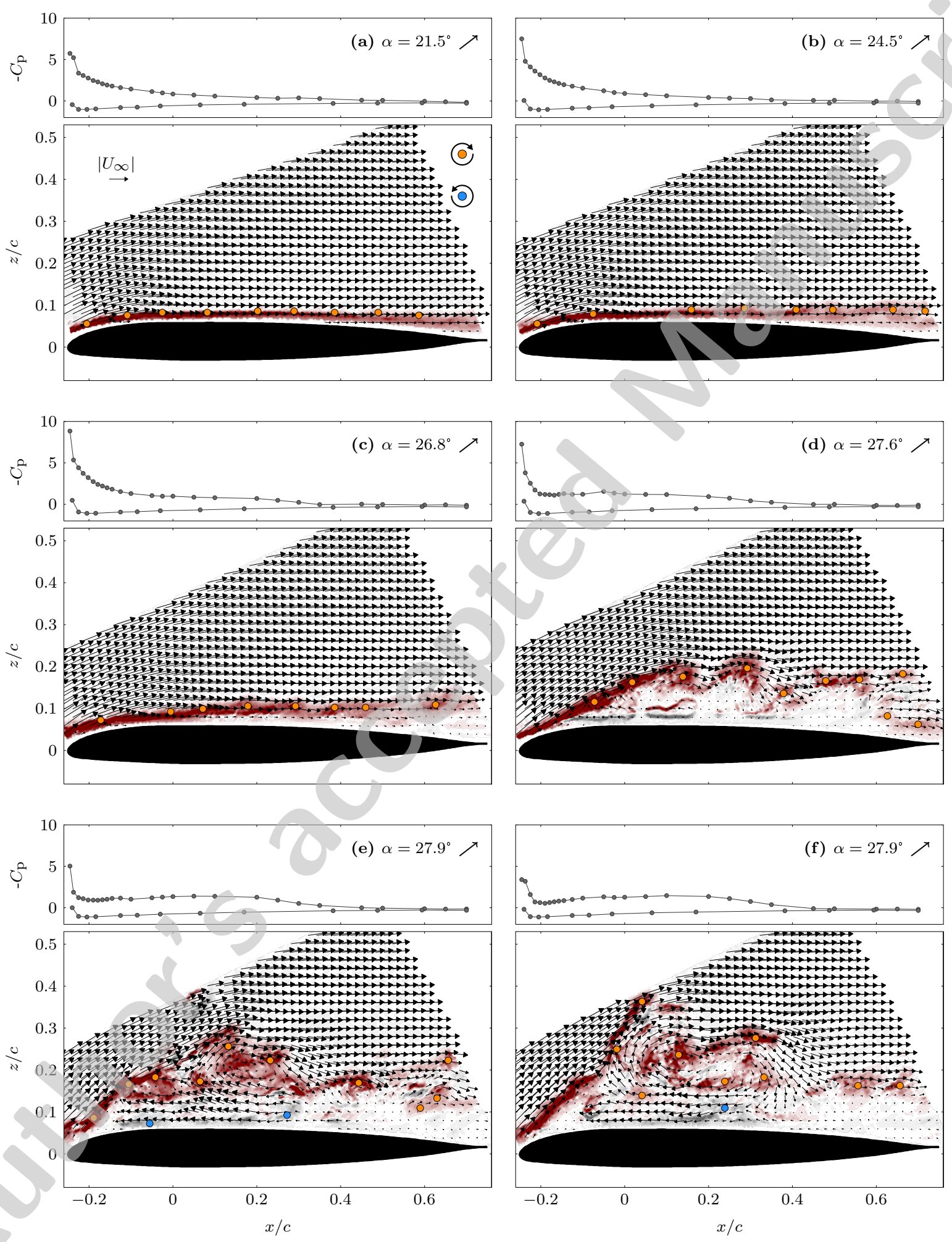

Fig. 3 For caption, see following page. 
8

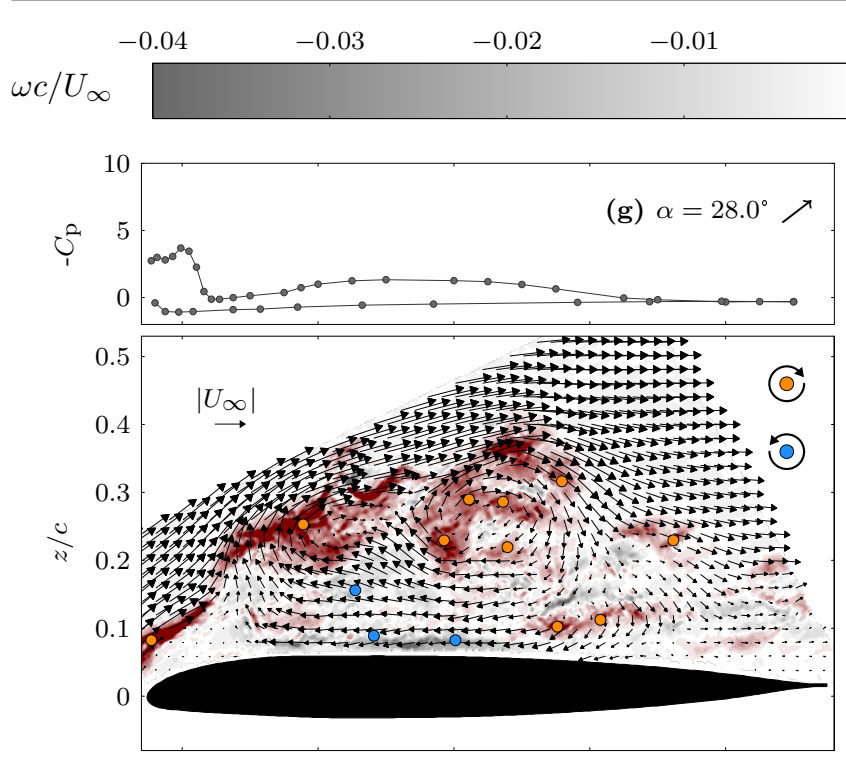

0.01

0.02

0.03

0.04
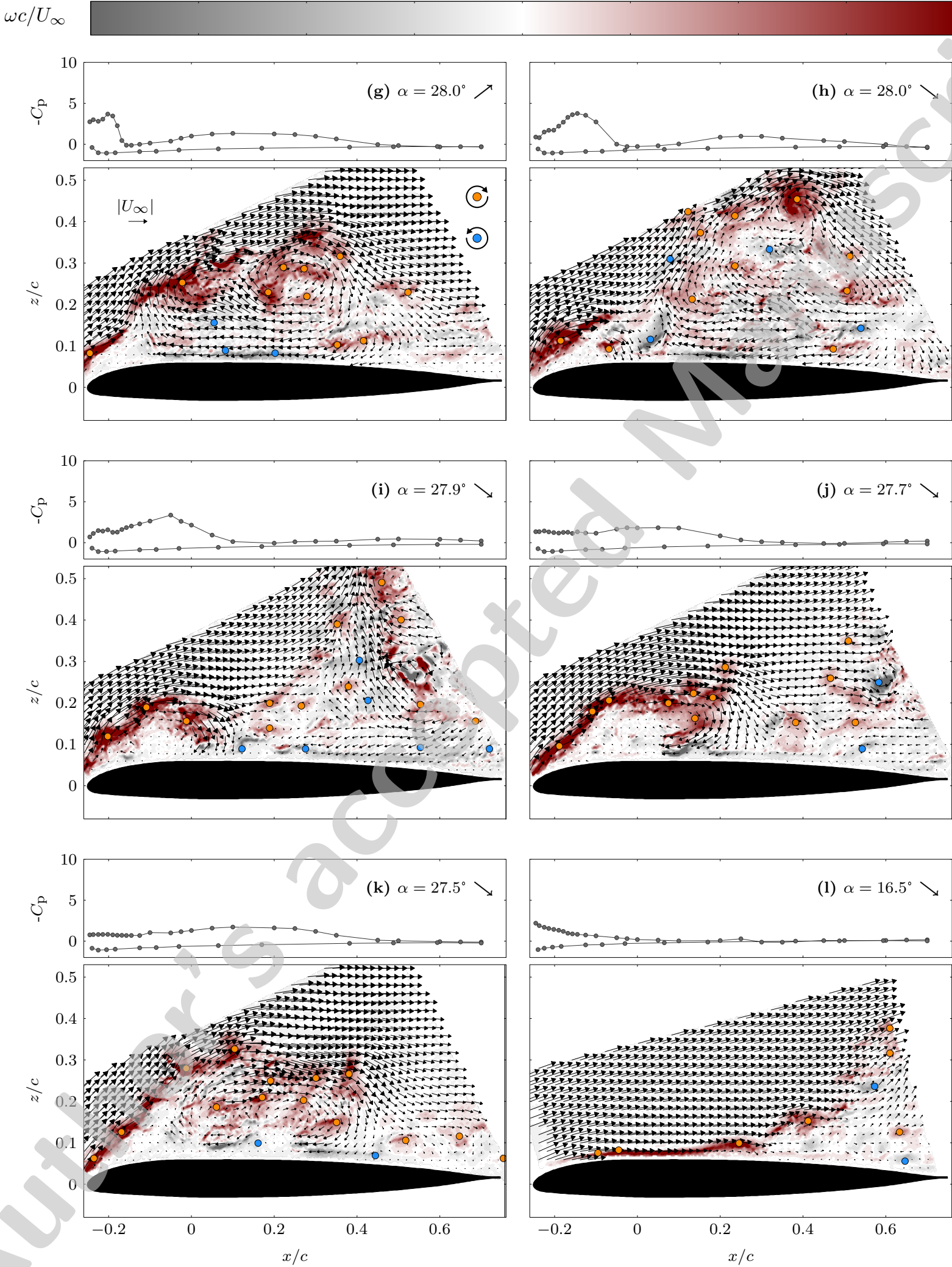

Fig. 3 Instantaneous flow fields with detected vortex cores: $(\bullet)$ clockwise and $(\bullet)$ anticlockwise rotation; and the respective surface pressure distribution for the states marked in figure 2 within a single oscillation $\left(\alpha_{0}=20^{\circ}, \alpha_{1}=8^{\circ}\right.$, and $\left.k=0.10\right)$. Only every sixth velocity vector is shown for the sake of visibility and the dimensionless out-of-plane component of the vorticity is colour-coded. 
flow reversal, the external flow is only mildly deviated while the aerodynamic lift keeps augmenting. This differs significantly from the static reference case, where flow reversal elicits massive flow separation accompanied by a dramatic decrease of the lift and pitching moment.

The flow field in figure 3(c) is not stable; the viscous interactions between the shear layer vortices rapidly increase and lead to merging of the small-scale structures, hence the occurrence of a secondary instability of the shear layer (see figure 3(d)). This roll-up process eventually coalesces in the formation of one large-scale vortical structure which is referred to as the primary dynamic stall vortex (figure 3(e)). This dynamic stall vortex thus consists of a combination of the rolled-up shear layer and the remnants of several vortices generated by a primary instability of the shear layer. Its presence is associated with a plateau in the surface pressure distribution between approximately $x=-0.1 c$ and $x=0.2 c$ on the suction side (figure 3(e)-(f)).

While the dynamic stall vortex continues to take up vorticity, counter-rotating vortices emerge near the airfoil's surface as a result of increasingly strong interactions between the stall vortex and the reversing flow (see figure $3(\mathrm{~g})$ ). The clockwise rotating primary stall vortex pushes the anticlockwise rotating structures towards the leading edge, thereby forcing itself to detach; this is known as vortex induced separation (cf. Peridier et al 1991; Obabko and Cassel 2002).

The detachment of the primary stall vortex marks the dynamic stall onset; it is the end of the stall development stage and the beginning of the stalled stage. With the primary stall vortex living its life, additional circulation accumulates upstream of it, leading to the formation of a secondary stall vortex. These primary originating vortices detach in close succession and are simultaneously convected downstream. During the convection process the flow can briefly follow the airfoil contour again. The massive flow separation downstream of approximately $x=0.1 c$ slows down the incoming flow and thus the convection of vorticity from the leading edge. This results in the formation of a leading edge recirculation region which is confined by a shear layer containing the accumulated vorticity (figure 3(h)$(\mathrm{k}))$. While this recirculation region grows and moves slowly downstream the confining shear layer rolls up to form anew a large-scale coherent structure. This process of yortex formation and detachment is repeatedly observed during downstroke. The large-scale vortex shedding and the associated large fluctuations of the aerodynamic load coefficients characterise the stalled stage. It continues until the flow reattaches close to the end of the downstroke (figure 3(1)). Finally, flow reat- tachment allows for the aerodynamic loads to return to their initial un-stalled values.

The succession of events elucidated above, is qualitatively observed for the entire parameter span relevant to this study. Hence, it is deemed to adequately cover the prominent events of the dynamic stall process for the prevailing flow conditions and chosen airfoil profile.

\subsection{Dynamic Stall Onset}

The prominent feature of the stall development stage is the formation and growth of a large-scale dynamic stall vortex which is pinched off from the airfoil's surface as a result of a vortex induced separation process. The dynamic stall onset is defined here as the detachment of the primary stall vortex which is determined from the experimental data based on the POD of the
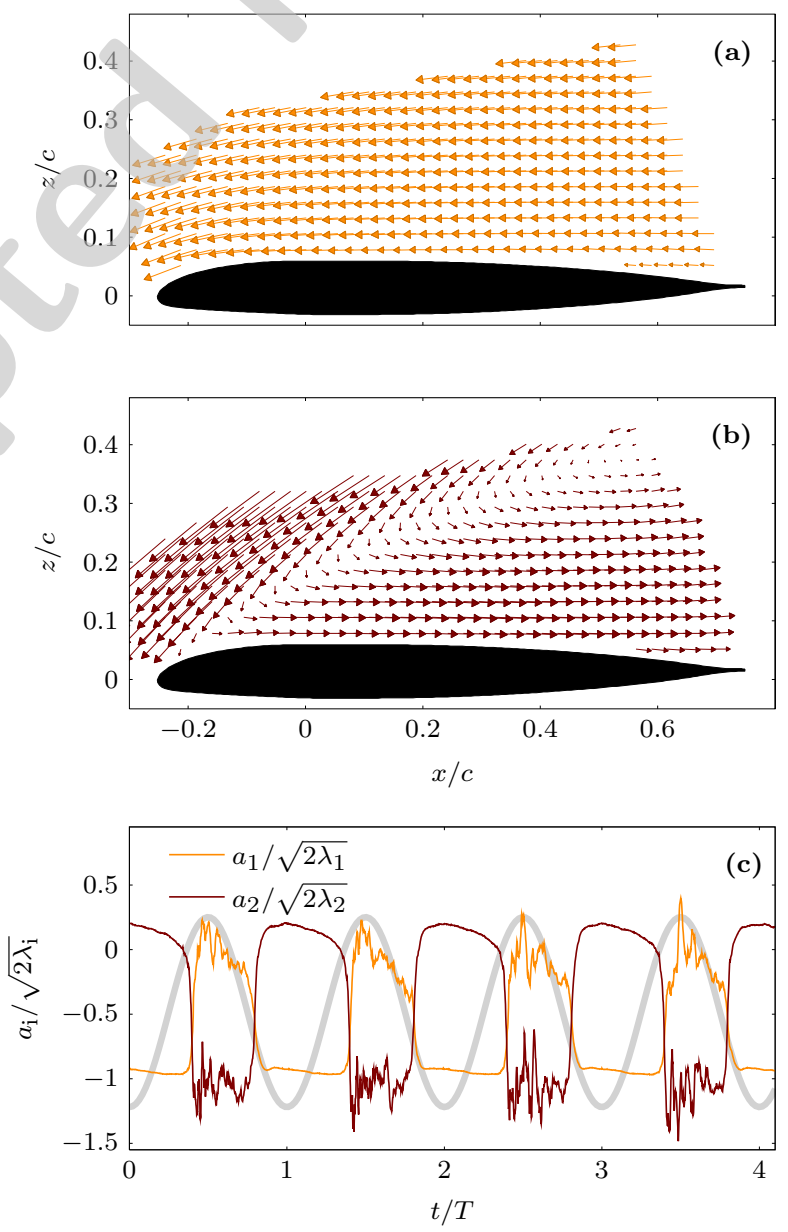

Fig. 4 POD modes: (a) the first and (b) second spatial modes $\psi_{\mathrm{i}}(x, z)$ and (c) the corresponding time development coefficient $a_{\mathrm{i}}(t)\left(\alpha_{0}=20^{\circ}, \alpha_{1}=8^{\circ}, k=0.05\right)$. The airfoil's oscillating motion is drawn auxiliary for four oscillation periods $T$. 
Table 1: Overview of the calculated dynamic stall quantities for the different oscillation parameter combinations considered.

\begin{tabular}{cccccc}
\hline$\dot{\alpha}_{\mathrm{ss}} c / U_{\infty}$ & $r_{\mathrm{eq}}{ }^{*}$ & $\alpha_{\mathrm{ds}}\left[^{\circ}\right]$ & $\Delta t_{\mathrm{ds}} U_{\infty} / c$ & $\alpha_{\mathrm{ds}}{ }^{\S}\left[^{\circ}\right]$ & $\Delta t_{\mathrm{ds}} U_{\infty} / c^{\S}$ \\
\hline $8.44 \times 10^{-3}$ & $5.24 \times 10^{-3}$ & $24.008(6)$ & $9.7(4)$ & $23.8(1)$ & $7.5(8)$ \\
$9.97 \times 10^{-3}$ & $5.24 \times 10^{-3}$ & $25.51(9)$ & $9.5(4)$ & $24.828(2)$ & $7.1(7)$ \\
$1.02 \times 10^{-2}$ & $5.24 \times 10^{-3}$ & $26.2(1)$ & $8.9(3)$ & $25.17(6)$ & $6.7(1)$ \\
$1.24 \times 10^{-2}$ & $6.98 \times 10^{-3}$ & $25.69(6)$ & $8.6(2)$ & $25.1(1)$ & $6.6(4)$ \\
$1.35 \times 10^{-2}$ & $6.98 \times 10^{-3}$ & $26.5(1)$ & $7.9(3)$ & $25.59(6)$ & $6.1(1)$ \\
$1.37 \times 10^{-2}$ & $6.98 \times 10^{-3}$ & $26.99(7)$ & $7.6(1)$ & $25.77(6)$ & $5.76(9)$ \\
$1.53 \times 10^{-2}$ & $7.85 \times 10^{-3}$ & $25.96(2)$ & $8.3(2)$ & $25.3(1)$ & $5.7(3)$ \\
$2.02 \times 10^{-2}$ & $1.05 \times 10^{-2}$ & $25.87(6)$ & $7.6(3)$ & $25.65(9)$ & $5.0(3)$ \\
$2.08 \times 10^{-2}$ & $1.05 \times 10^{-2}$ & $27.58(7)$ & $6.6(2)$ & $26.47(9)$ & $4.8(1)$ \\
$2.08 \times 10^{-2}$ & $1.05 \times 10^{-2}$ & $27.4(1)$ & $6.6(3)$ & $26.4(1)$ & $4.9(2)$ \\
$2.52 \times 10^{-2}$ & $1.40 \times 10^{-2}$ & $25.77(5)$ & $6.8(1)$ & $25.78(5)$ & $4.5(2)$ \\
$2.74 \times 10^{-2}$ & $1.40 \times 10^{-2}$ & $27.88(4)$ & $6.3(2)$ & $26.9(1)$ & $4.3(2)$ \\
$2.78 \times 10^{-2}$ & $1.40 \times 10^{-2}$ & $28.9(1)$ & $5.7(2)$ & $27.4(1)$ & $4.1(1)$ \\
\hline
\end{tabular}

The numbers in between the brackets indicate the standard deviation of the data.

* Equivalent reduced pitch rate as defined by Sheng et al (2008).

$\S$ Stall onset determined as the point at which the maximum leading edge suction peak is achieved.
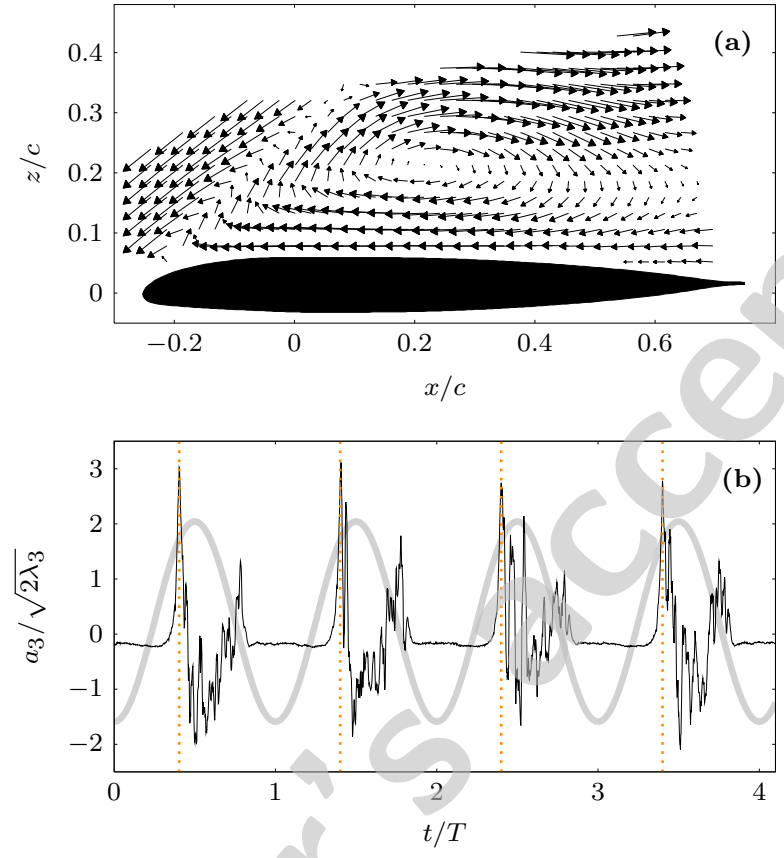

Fig. 5 (a) The third spatial POD mode $\psi_{3}(x, z)$ and (b) the corresponding time development coefficient $a_{3}(t)\left(\alpha_{0}=20^{\circ}, \alpha_{1}=\right.$ $\left.8^{\circ}, k=0.050\right)$.

two-component velocity field $\mathbf{u}=(u, w)$ (equation 2). The spatial modes respective the velocity decomposition are denoted by $\psi_{\mathrm{i}}(x, z)$ and their temporal evolution and relative contribution are indicated by the time development coefficients $a_{\mathrm{i}}(t)$.

The first and second mode represent a fully attached and a fully separated flow state respectively (see fig- ure 4(a)-(b)). Within a dynamic stall life cycle the flow generally evolves from attached to massively separated during the upstroke and - accompanied by large hysteresis - to reattached again near the end of the downstroke. Hence, during most of the cycle, i. e. except for the transient stages, the flow can be considered either attached or separated and it is not surprising that the - in a statistical sense - dominant modes of the POD represent exactly these limiting states. The alternating dominance and associated transfer of energy from one limiting state into the other is clearly observable in figure 4(c) depicting the temporal evolution of the first and second mode coefficient. However, the most interesting mode with regard to the stall onset is the third spatial mode (see figure 5(a)). This mode can be interpreted as a large-scale coherent structure or dynamic stall vortex, an idea that is supported by the temporal development of the coefficient $a_{3}(t)$ (figure $5(\mathrm{~b})$ ). During the first part of the cycle the flow is attached and the magnitude of $a_{3}(t)$ is small and approximately constant. Hence, the contribution of $\psi_{3}$ to the decomposition is inconsiderable and it only gains importance near the end of the upstroke motion when the primary stall vortex is formed. This primary large-scale structure dominates the flow field until it is pinched off from the separated boundary layer that provides its circulation. Consequentely, the weight of the third mode attains a local maximum shortly before the detachment of the primary stall vortex, i. e. stall onset. The angle of attack at this local maximum is referred to as the dynamic stall onset angle of attack and is denoted by $\alpha_{\mathrm{ds}}$. 

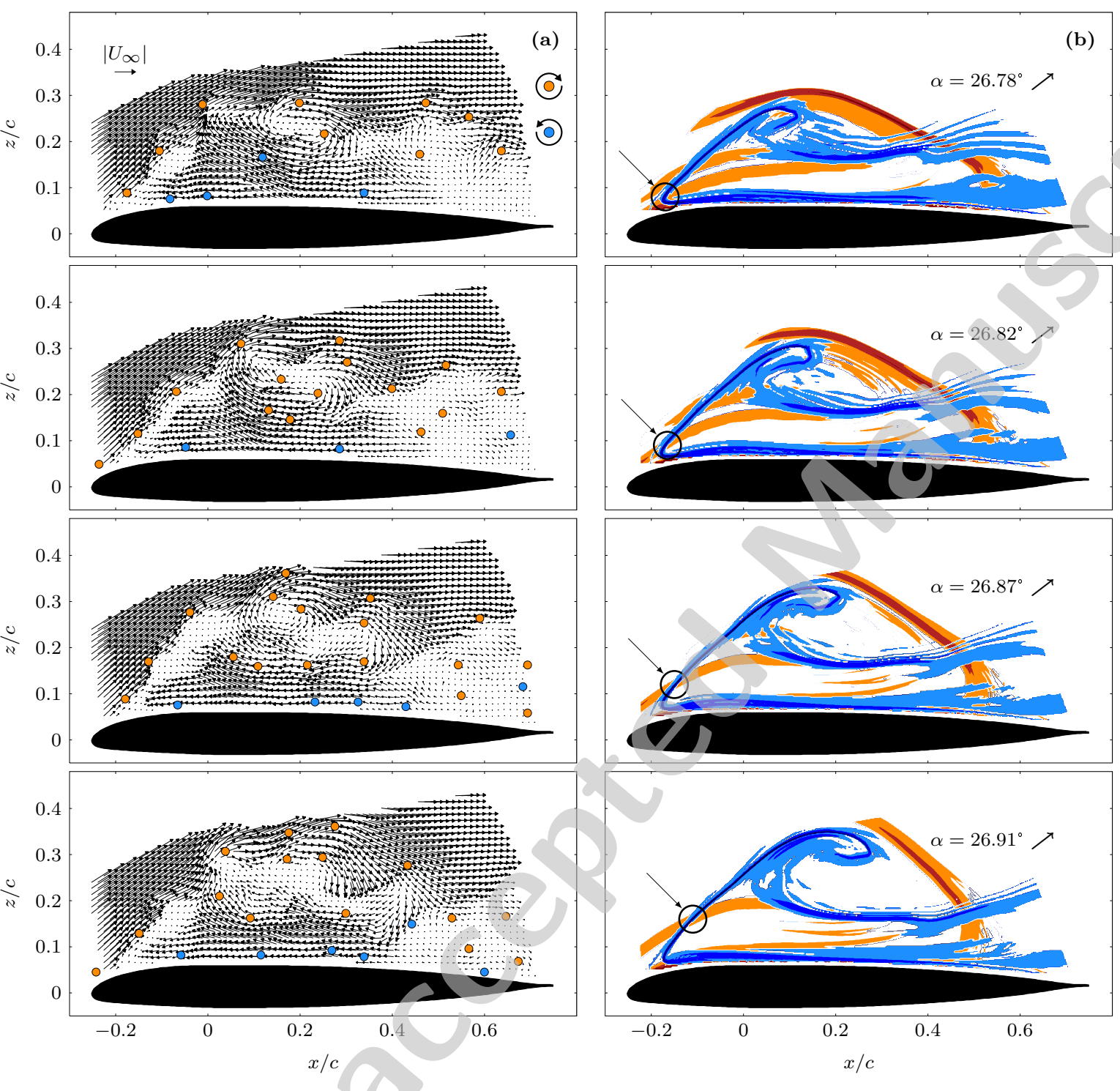

Fig. 6 Flow field topology and vortex distribution shortly after dynamic stall onset: (a) sequence of instantaneous velocity fields and the centres of $(\bullet)$ clockwise and $(\bullet)$ anticlockwise rotating vortices and $(b)$ the corresponding Lagrangian coherent structures indicated by the ridges in the pFTLE and nFTLE fields. The black circles mark the approximate position of the saddle point $\left(\alpha_{0}=20^{\circ}, \alpha_{1}=8^{\circ}\right.$, $k=0.05)$.

The fact that the POD based assignment of the stall onset indeed corresponds to an invasive topological change was verified by the Lagrangian coherent structure analysis. The LCSs for four instantaneous subsequent velocity fields shortly after dynamic stall onset are depicted in figure 6 . The flow field around the intersection of a repelling and an attracting material line, visualised by ridges in the pFTLE and nFTLE fields respectively, resembles that of a saddle point. This saddle point moves in time and indicates the detachment of the vortex from the boundary layer, which marks the end of the stall development stage. Furthermore, the comparison of the trajectories of the detected vortices, emerging before and after the assessed stall onset confirms that this is indeed a critical point. A clear change can be recognised in the orientation of the trajectories of the vortices that originate at the very leading edge for angles of attack before and beyond $\alpha_{\mathrm{ds}}$ (see figure 7).

Per se, POD modes are mathematical constructions; associating them with coherent flow structures should not be done without proper consideration. Nevertheless, due to the congruence with the Eulerian and Lagrangian picture, the POD method is found adequate 
Fig. 7 Trajectories of positive vortices emerging before and after stall onset. The histogram depicts the probability density functions of the vertical location of the vortex centres respective the different stages.

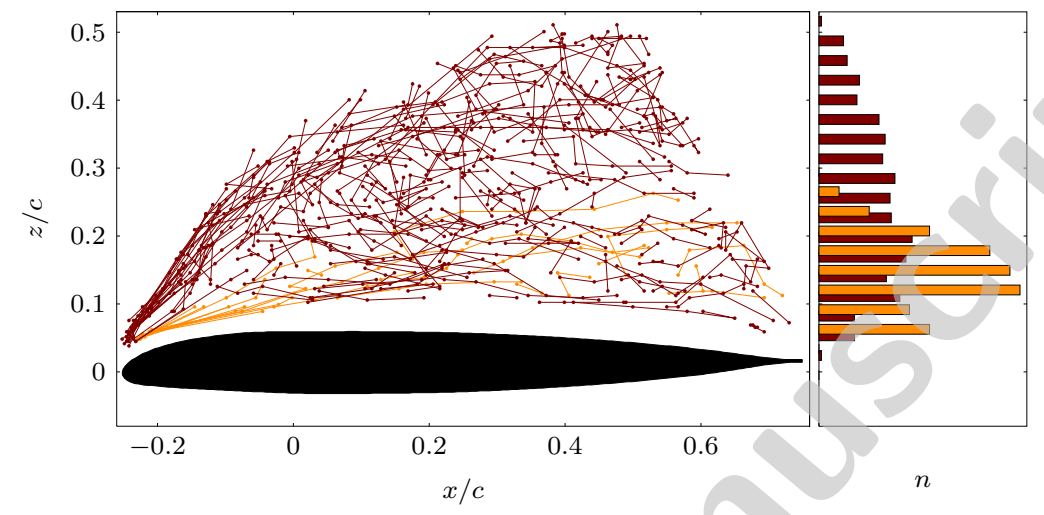

and practical for defining the dynamic stall onset in the present context. Moreover, it allows for pinpointing the actual instant at which the dynamic stall vortex separates. Per contra, stall angles determined based on anomalies in the airloads correspond to points that either indicate incipient stall or represent aftermath of the vortex' separation and its subsequent downsteam convection (Wilby 2001). Here, for example, the stall angle determined as the point at which the maximum leading edge surface pressure peak is achieved is consistently smaller than the stall angle specified based on the flow field observations (see table 1). An accurate characterisation of the different stages of the dynamic stall process of an airfoil, in particular of the stall onset, is highly desirable for improving semi-empirical dynamic stall models which approximate the key features of the process in a physically representative way, e.g. the Beddoes-Leishman model (Leishman and Beddoes 1989; Beddoes 1993).

\subsection{Dynamic Stall Delay}

Dynamic stall of an airfoil is governed by many parameters. Besides flow parameters such as Mach and Reynolds number, parameters describing the airfoil's motion also strongly influence the development of the stalling process. For a oscillating airfoil in a uniform flow at given Mach and Reynolds number, the prediction of the stall onset is essentially a three-parameter problem, with $\alpha_{0}, \alpha_{1}$, and $k$ being interrelated. Since the stall process is qualitatively similar for airfoils subjected to oscillating or ramp-type motions (McCroskey 1981), we seek for a single parameter to described the overall influence of the airfoil's unsteadiness on the stall onset.

Sheng et al (2008) introduced the equivalent reduced pitch rate $r_{\mathrm{eq}}=\alpha_{1} k$ for oscillating motions to reduced the number of parameters. According to this concept, stall onset is independent of $\alpha_{0}$ under deep stall conditions and increases linearly with $r_{\mathrm{eq}}$. For the experimental configurations considered here the dynamic stall onset and the corresponding time delay with respect to static stall are apparently not independent of $\alpha_{0}$ and a linear relationship between $\alpha_{\mathrm{ds}}$ and $r_{\mathrm{eq}}$ is not confirmed (see table 1). Alternatively, we introduce the instantaneous effective unsteadiness $\dot{\alpha}_{\text {ss }}$ as a single representative parameter to describe the influence of the airfoil's oscillating motion on the stall onset. The instantaneous effective unsteadiness is defined as the rate of change of $\alpha$ at $t=t_{\mathrm{ss}}$, which is the moment $\alpha_{\mathrm{ss}}$ is reached, and equals the reduced pitch rate for ramptype motions.

Considering the stall onset angle in function of the normalised instantaneous effective unsteadiness, an overall increase of $\alpha_{\mathrm{ds}}$ with $\dot{\alpha}_{\mathrm{ss}}$ is observed (figure 8). The angle of attack increases nonlinearly during the stall development stage and we should rather focus on its time scale given by the dynamic stall delay $\Delta t_{\mathrm{ds}}$.

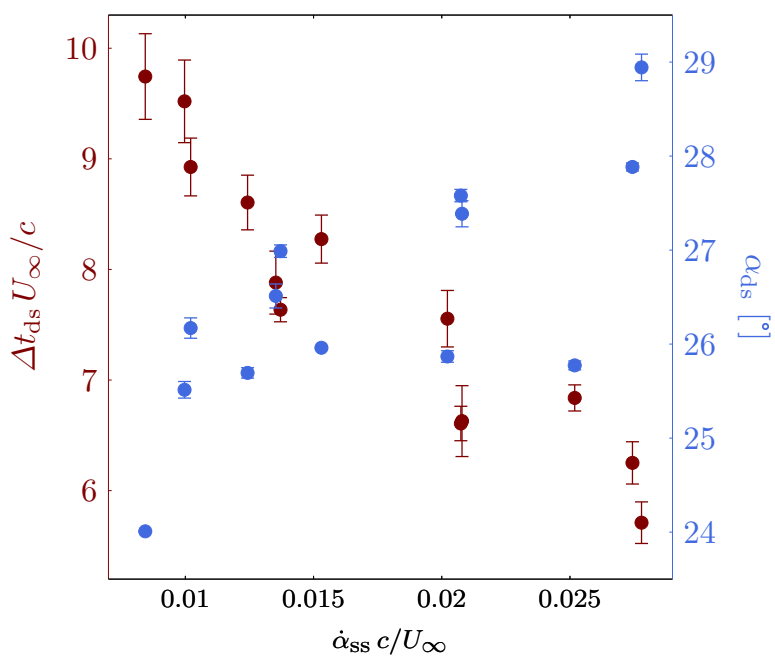

Fig. 8 Dynamic stall angle and delay versus normalised instantaneous effective unsteadiness. 
The stall delay decreases with increasing $\dot{\alpha}_{\mathrm{ss}}$ and dynamic stall is actually promoted rather than delayed by increasing unsteadiness.

\subsection{Light and Deep Dynamic Stall}

With regard to the separation behaviour of the subsonic flow around an oscillating airfoil two distinct stall regimes can be differentiated. According to the nomenclature of McCroskey, light and deep dynamic stall refer to the different stall regimes whose distinguishing attributes are summarised in figure 9. This classification is based on the degree and extent of the flow separation without specifying the underlying physical mechanisms. However, this information is essential in order to predict whether a particular oscillation will provoke light or deep dynamic stall. Attempting to address this issue, we consider the following parameter combinations:

(i) $\alpha_{0}=18^{\circ}, \alpha_{1}=6^{\circ}$, and $k=0.10$;

(ii) $\alpha_{0}=20^{\circ}, \alpha_{1}=6^{\circ}$, and $k=0.10$.

where the former is a example of a light stall case and the latter is representative for a deep stall case based on observations of the flow field and the mean lift coefficient hysteresis curves (figure 10).

The discrepancy in the extent of the separation region for both cases can be clearly observed in figure 11 where the trajectories of all positive vortices emerging within different parts of the oscillation cycle, at the end of the upstroke and at the beginning of the downstroke

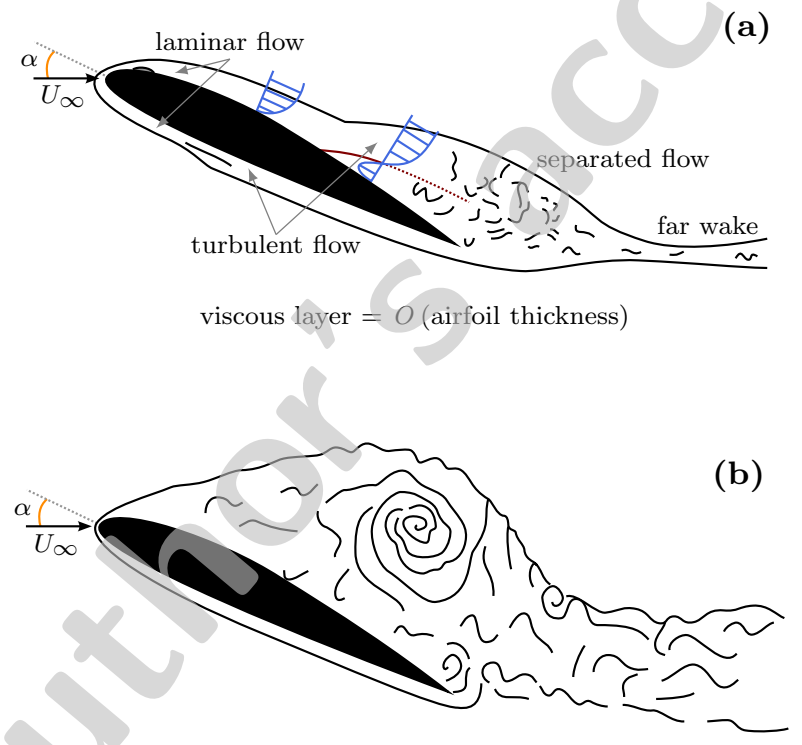

viscous layer $=O($ airfoil chord $)$

Fig. 9 Topology of a (a) light and (b) deep stall configuration (from McCroskey and Pucci (1982)).

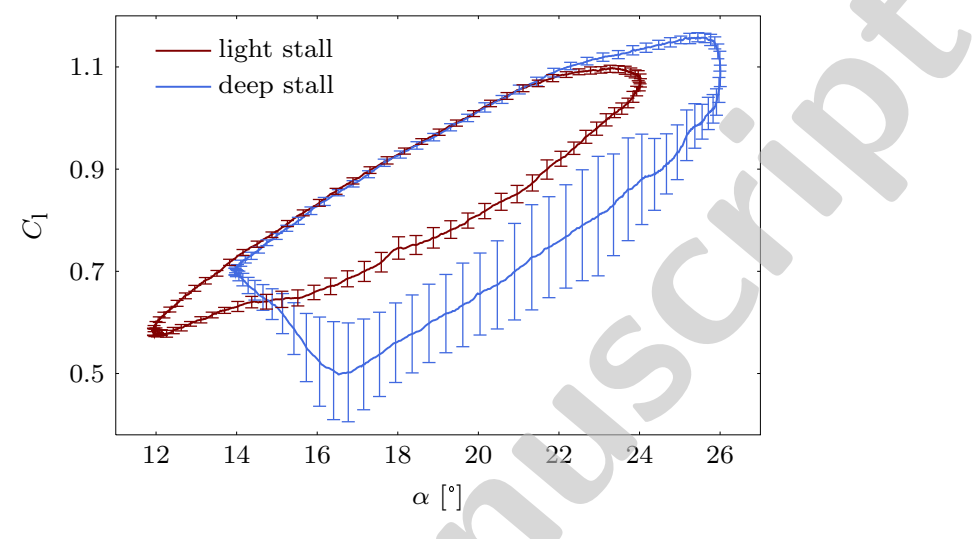

Fig. 10 Average lift coefficient hysteresis for a light stall $\left(\alpha_{0}=\right.$ $\left.18^{\circ}, \alpha_{1}=6^{\circ}, k=0.10\right)$ and a deep stall configuration $\left(\alpha_{0}=20^{\circ}\right.$, $\left.\alpha_{1}=6^{\circ}, k=0.10\right)$. Error bars indicate the standard deviation.

are depicted together with the vertical distribution of the vortex positions.

For the deep stall case with maximum angle of attack $\alpha_{\max }=26^{\circ}$, the dynamic stall onset angle of attack is determined to be $\alpha_{\mathrm{ds}}=25.8(1)^{\circ}$ during upstroke. Massive flow separation is initiated on the upstroke giving rise to a large separated region whose vertical extent is of the order of the airfoil chord (figure 11(b) left and histogram).

For the light stall case on the other hand, the maximum angle of attack is lower $\left(\alpha_{\max }=24^{\circ}\right)$ and the primary stall vortex is not yet fully developed before the downstroke motion is initiated. The oscillation direction is changed before the dynamic stall onset angle is reached and the primary stall vortex is not pinched off and ejected away as a result of viscous interactions but is forced to separate by the reversing oscillation direction. Due do downward entrainment - as a result of the airfoil's downstroke motion - the vortical structures emerging near the airfoil's surface tend to follow the contour briefly before detaching yielding a smaller separation region, whose height is of the order of the airfoil thickness, congruent with the light stall regime. Furthermore, the vortex formation process of the primary stall vortex is prematurely terminated and separation is enforced yielding a weaker vortex. As a consequence, the fluctuations of the lift coefficient in the light stall case are less pronounced (cf. figure 10). The phase angle of the oscillation at which the dynamic stall onset is observed, fundamentally influences the extent and duration of the separation phenomenon.

Reverting to these observations, an endorsement of McCroskey's classification of dynamic stall regimes is proposed here. The idea is to distinguish between light or deep stall based on the onset of dynamic stall. More precisely, a particular parameter combination yields a 

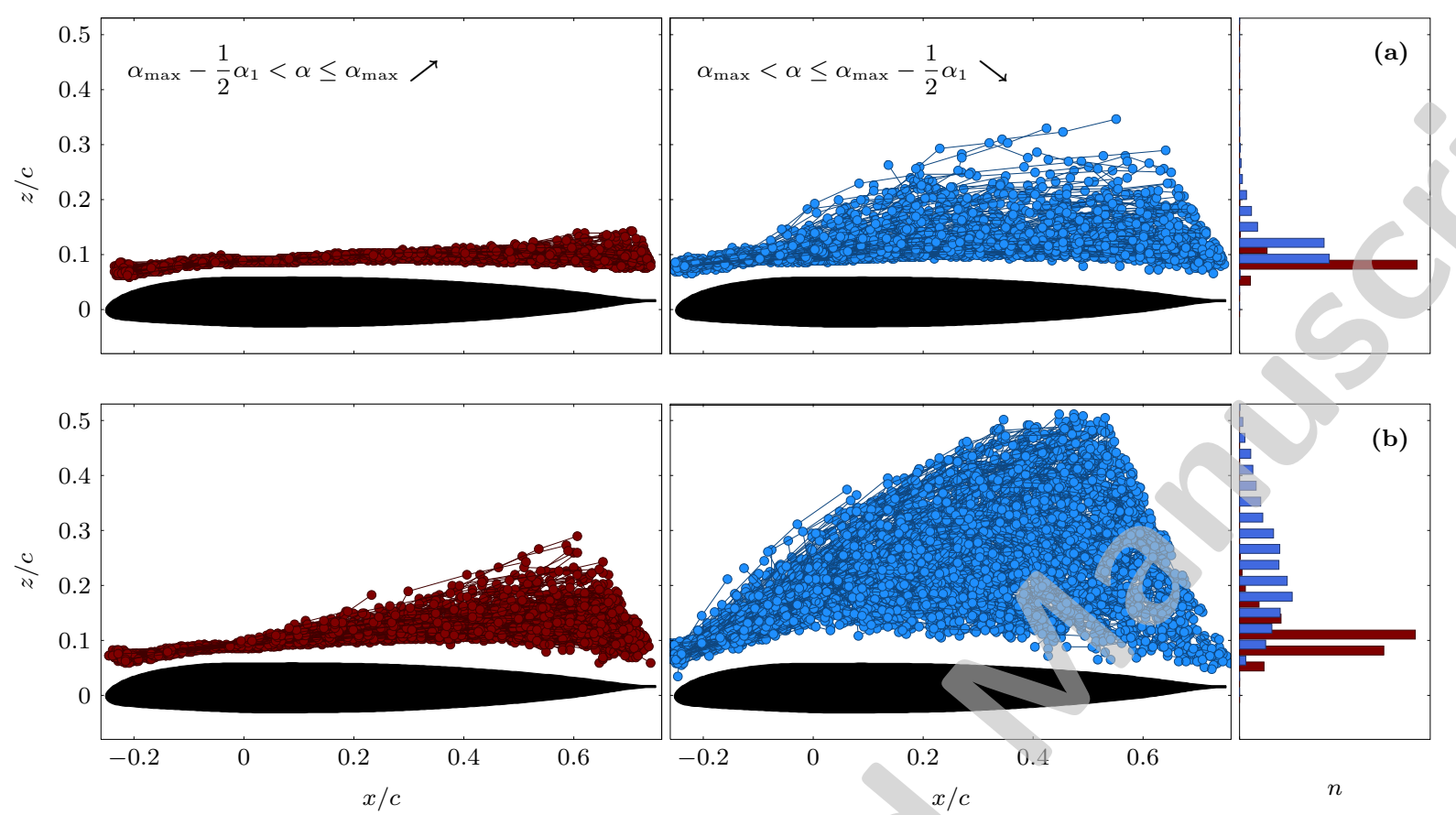

Fig. 11 Trajectories of positive vortices emerging for a light and deep stall configuration within different parts of the oscillation cycle: (a) at the near of the upstroke and (b) at the beginning of the downstroke. The histograms depict the probability density functions of the vertical location of the vortex centres for the different phase intervals.

dynamic stall regime that is referred to as light dynamic stall when the downstroke motion is set in before the stall development is completed and the separation of the primary stall vortex is forced to occur at the top of the cycle. Vice versa, a deep dynamic stall regime is encountered when dynamic stall onset occurs during the upstroke part of the airfoil's motion.

\section{Conclusion and Perspectives}

The characteristic features of the unsteady flow field were identified and analysed utilising various coherent structure identification methods. The combination of time-resolved imaging and an extensive coherent structure analysis allowed for analysis of the dynamics and interaction of vortical structures. The unsteady flow development within a single dynamic stall life cycle was classified into five successive stages. The dynamic stall onset was identified as a result of a vortex induced separation and was specified directly from the velocity field. A representative description of the influence of the airfoil's motion on the stall onset was found in terms of the rate of change of the angles of attack at the moment when the static stall angle is exceeded, denoted by the instantaneous effective unsteadiness. Dynamic stall onset was found to be promoted by increasing unsteadi- ness. When the onset of dynamic stall on an oscillating airfoil occurred before reaching the maximum angle of attack was reached, the flow was found to share the general features of deep dynamic stall. A light dynamic stall regime on the other hand was encountered when the oscillation direction was inverted before the stall onset angle of attack was reached.

Due to the inherent unsteady nature of the dynamic stall process, the time-resolved approach is preferential. In continuation, future research will focus on: characterising the shear layer development within the stall development stage; correlating the passage of coherent structures in the separated flow region and fluctuations of the aerodynamic load coefficients; and identifying the differences and resemblances of the dynamic and static stall development. Based on the new stall onset specification, a modification of empirical stall models is envisaged.

Acknowledgements The authors thank H. Mai, T. Büte, J. Nuhn and A. Henning for their contribution to the wind tunnel measurements and K. Kindler for comments.

\section{References}

Aubry N, Guyonnet R, Lima R (1991) Spatiotemporal analysis of complex signals: Theory and applications. J Stat Phys 
64:683-739

Beddoes T (1993) A third generation model for unsteady aerodynamics and dynamic stall. RP 908, Westland Helicopter Limited

Berkooz G, Holmes P, Lumley J (1993) The propor orthogonal decomposition in the analysis of turbulent flows. Ann Rev Fluid Mech 25:539-575

Carr L (1988) Progress in analysis and prediction of dynamic stall. J Aircraft 25(1):6-17

Carr L, McAlister K, McCroskey W (1977) Analysis of the development of dynamic stall based on oscillating airfoil experiments. TN D-8382, NASA

Chakraborty P, Balachandar S, Adrian R (2005) On the relationship between local vortex identification schemes. J Fluid Mech 535:189-214

Chong M, Perry A, Cantwell B (1990) A general classification of three-dimensional flow fields. Phys Fluids A: Fluid Dyn $2: 765-777$

Cucitore R, Quadrio M, Baron A (1999) On the effectivenss and limitations of local criteria for the identification of a vortex. Eur J Mech B Fluids 18(2):261-282

Garth C, Gerhardt F, Tricoche X, Hagen H (2007) Efficient computation and visualization of coherent structures in fluid flow applications. IEEE Trans Visual Comput Graphics 13(6):1464-1471

Graftieaux L, Michard M, Grosjean N (2001) Combining PIV, POD and vortex identification algorithms for the study of unstready turbulent swirling flows. Meas Sci Technol 12:14221429

Haller G (2001) Distinguished material surfaces and coherent structures in three-dimensional fluid flows. Physica D 149:248-277

Haller G (2002) Lagrangian coherent structures from approximate velocity data. Phys Fluids 14(6):1851-1861

Haller G (2005) An objective definition of a vortex. J Fluid Mech 525:1-26

Ho C, Huerre P (1984) Perturbed free shear layer. Ann Rev Fluid Mech 16:365-424

Hunt J, Wray A, Moin P (1988) Eddies, stream, and convergence zones in turbulent flows. In: Proceedings of the Summer Program 1988, N89-24555, pp 193-208

Jeong J, Hussain F (1995) On the identification of a vortex. J Fluid Mech 285:69-94

Leishman J, Beddoes T (1989) A semi-empirical model for dynamic stall. J Am Helicopter Society 34(3):3-17

Lekien F, Coulliette C (2001-2002) Mangen software package. URL http://www.lekien.com/ francois/software/mangen/

Lugt H (1979) Recent Developments in Theoretical and Experimental Fluid Dynamics, Springer, chap The Dilemma of Defining a Vortex, pp 309-321

Lumley J (1970) Stochastic Tools in Turbulence. Applied Mathematics and Mechanics - An International Series of Monographs 12, Academic Press

McAlister K, Carr L, McCroskey W (1978) Dynamic stall experiments on the NACA0012 airfoil. TP 1100, NASA

McCroskey W (1981) The phenomenon of dynamic stall. TM 81264, NASA

McCroskey W, Pucci S (1982) Viscous-inviscid interactions on oscillating airfoils in subsonic flow. AIAA J 20(2):167-174

Michard M, Graftieaux L, Lollini L, Grosjean N (1997) Identification of vortical structures by a non local criterion - application to PIV measurements and DNS-LES results of turbulent rotating flows. In: Proceedings of the $11^{\text {th }}$ Conference on Turbulent Shear Flows, Grenoble, France

Obabko A, Cassel K (2002) Detachement of the dynamic stall vortex above a moving surface. AIAA J 40(9):1811-1822
Peacock T, Dabiri J (2010) Introduction to focus issue: Lagrangian coherent structures. Chaos 20:1-3

Peridier V, Smith F, Walker J (1991) Vortex-induced boundary layer separation. part 1. the unsteady limit problem $R e \rightarrow \infty$. J Fluid Mech 232:99-131

Richard H, Bosbach J, Henning A, Raffel M, van der Wall B (2006) 2C and 3C PIV measurements on a rotor in hover condition. In: Proceedings of the $13^{\text {th }}$ International Symposium on Applications of Laser Techniques to Fluid Mechanics, Lisbon, Portugal

Robinson S (1991) Coherent motions in the turbulent boundary layer. Ann Rev Fluid Mech 23:601-639

Shadden S, Lekien F, Marsden J (2005) Definition and properties of Lagrangian coherent structures from finite-time lyapunov exponents in two-dimensional aperiodic flows. Physica D 212:271-304

Sheng W, Galbraith RM, Coton F (2006) A new stall-onset criterion for low speed dynamic stall. J Sol Energy Eng 128:461471

Sheng W, Galbraith R, Coton F (2008) Prediction of dynamic stall onset for oscillatory low-speed airfoils. J Fluids Eng 130:101,204-1-101,204-8

Shih C, Lourenco L, Van Dommelen L, Krothapalli A (1992) Unsteady flow past an airfoil pitching at constant rate. AIAA J 30(5):1153-1161

Sirovich L (1987) Turbulence and the dynamics of coherent structures; part I, II and III. Q Appl Math 45(3):561-590

Wilby P (2001) J Am Helicopter Society 46(3):210-220

Wu J, Ma H, Zhou M (2006) Vorticity and Vortex Dynamics. Springer 